\title{
钯催化甲基苯基膦氢的不对称烷基化反应合成含膦手性中心化合物
}

\author{
李 春 ${ }^{a}$ 李伟逊 ${ }^{b}$ 许 胜*,a 段伟良*,b \\ ( ${ }^{a}$ 华东理工大学化学与分子工程学院 上海 200237) \\ ( ${ }^{b}$ 中国科学院上海有机化学研究所金属有机化学国家重点实验室 上海 200032)
}

\begin{abstract}
摘要 研究了 Pincer-钯催化的甲基苯基膦氢对卤代烃的不对称取代反应. 以高收率、中等的立体选择性得到含膦手性 中心的膦化合物, 同时对反应的催化循环和可能的立体化学过程进行了探讨.

关键词＼cjkstart膦手性化合物; Pincer-钯催化剂; 甲基苯基膦氢; 不对称烷基化
\end{abstract}

\section{Pd-Catalyzed Asymmetric Alkylation of Methylphenylphosphine with Alkyl Halides for the Synthesis of P-stereogenic Compounds}

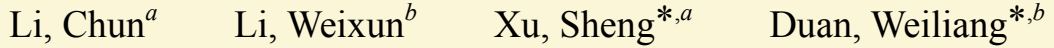 \\ ( ${ }^{a}$ School of Chemistry \& Molecular Engineering, East China University of Science and Technology, Shanghai 200237) \\ ( ${ }^{b}$ State Key Laboratory of Organometallic Chemistry, Shanghai Institute of Organic Chemistry, \\ Chinese Academy of Sciences, Shanghai 200032)
}

\begin{abstract}
A bis(phosphine) (PCP) pincer palladium-catalyzed asymmetric alkylation of methylphenylphosphine with alkyl halides was developed for the synthesis of P-chiral phosphorus compounds in mild conditions. The corresponding products were obtained in high yields with moderate enantioselectivities. The possible catalytic cycle and stereochemical pathway have also been proposed via the Pincer-PdPMe $(\mathrm{Ph})$ intermediate, which is generated from the reaction of Pd catalyst with methylphenylphosphine.
\end{abstract}

Keywords P-stereogenic compound; Pincer-Pd catalyst; methylphenylphosphine; asymmetric alkylation

含膦手性中心膦化合物是一类重要的配体, 可被应 用于各种不对称催化反应之中 ${ }^{[1]}$. 最早由 Horner 等 ${ }^{[2]}$ 于 1961 拆分制备得到, 其后 Knowles 和 Horner 等分别使 用膦手性化合物应用于烯烃的均相不对称氢化之中 ${ }^{[3]}$, 开启了不对称氢化反应研究的序幕. 但相对于随后发展 的各种含碳手性骨架膦配体(如 DIOP, Duphos 等)或轴 手性骨架的膦配体 (如 BINAP 等) 而言, 膦手性中心配体 在不对称催化中的应用还不够广泛, 主要的原因是由于 它们的合成更为复杂, 不易制备获得 ${ }^{[4]}$. 传统的合成膦 手性化合物的方法多基于使用当量手性试剂对外消旋 膦化合物的拆分 ${ }^{[1]}$. 使用不对称催化方法合成膦手性化 合物是一条更为直接的途径, 近年来已有一定报道. 主 要集中在前手性膦化合物的去对称化 ${ }^{[5]}$, 膦氢化合物对 不饱和双键/三键的加成 ${ }^{[6]}$, 以及对卤代烃的偶联 ${ }^{[7]}$ 或取
代反应之中 ${ }^{[8]}$. 对于膦氢化合物与卤代烃的不对称烷基

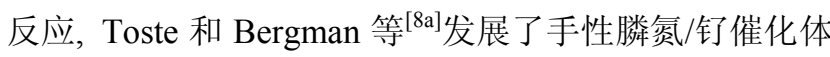
系应用于甲基苯基膦氢的烷基化反应，取得了不错的结 果(up to $85 \% e e$ ). 与此同时 Glueck 等使用手性双膦-铂 金属催化大位阻二级膦烷(2,4,6-三异丙基苯基甲基膦 氢)对卤代烃的亲核取代反应，立体选择性也可达 $81 \%{ }^{[8 b]}$. 近来我们小组 ${ }^{[9]}$ 集中研究了钯催化二芳基膦氢 化合物对缺电子烯烃的不对称加成反应，得到了各种高 光活纯度的含碳手性中心的膦化合物. 我们推测当二芳 基膦上的取代基不相同时：譬如苯基甲基膦氢，与亲电 试剂反应, 可以生成含膦手性中心的膦化合物. 在此, 我们报道钯催化甲基苯基膦氢对卤代烃的不对称取代 反应合成膦手性化合物的初步结果.

* E-mail: wlduan@mail.sioc.ac.cn; Tel.: 021-54925203; Fax: 021-64166128

Received January 21, 2013; revised February 26, 2013; published online March 1, 2013.

Project supported by the National Basic Research Program of China (973 Program, 2010CB833300), the National Natural Science Foundation of China (Nos. 20902099, 21172238), and the Found of Shanghai Institute of Organic Chemistry, Chinese Academy of Sciences.

国家重点基础研究发展计划(973 计划, No. 2010CB833300)、国家自然科学基金(Nos. 20902099, 21172238)、中国科学院上海有机化学研究所启动经 费资助项目. 


\section{2 结果与讨论}

\section{1 烷基化反应条件的优化}

甲基苯基膦氢与苄氯被使用作为标准底物来测试 催化剂的效果, 由于产物三价膦易被氧化, 为方便分离 纯化, 反应结束时加入嗍烷二甲硫醚溶液, 分离得到三 价膦一嗍烷络合物. 我们首先使用小组先前合成的催化 剂 $(S, S)-4^{[9 a, 10,11]}$, 以二氯甲烷为溶剂时, 仅取得 $32 \%$ 的 立体选择性(表 1, Entry 1). 使用手性氨基醇衍生, 相对 较容易制备的 NCN 钳形钯催化剂 5 , 仅得 $24 \%$ 收率的消 旋产物(表 1, Entry 2). 于是我们就使用催化剂 $\mathbf{4}$ 对溶剂 进行了篮选. 结果表明室温下 DMF 相对于其他各种溶 剂有最高为 $45 \%$ 的立体选择性(表 1, Entries 3 11). 对 碱的篎选表明, 叔丁醇钾和苯酚钠给出消旋产物, 而有 机碱如 DIPEA, 三乙胺获得类似于碳酸钾的对映选择 性, 但收率较低(表 1, Entries 12 16). 最后, 降低温度 的实验表明: $-30{ }^{\circ} \mathrm{C}$ 时可以 $92 \%$ 收率, $53 \% e e$ 得到产 物, $-55{ }^{\circ} \mathrm{C}$ 时, 虽然 $e e$ 值可提高至 $59 \%$, 但收率低于 10\% (表 1, Entries 17 18). 不加钯催化剂时背景反应表 明， $-30{ }^{\circ} \mathrm{C}$ 时, 苯基甲基膦氢与苄氯的反应反应进行 很慢(表 1, Entry 19). 我们最终选择了 DMF 作为溶剂, 碳酸钾作为碱, $-30{ }^{\circ} \mathrm{C}$ 下反应为最优条件.

\section{2 烷基化反应的底物拓展}

在确定最终的反应条件之后, 接下来我们对底物的 普适性进行了拓展和研究. 对于氯化苄之类的底物, 芳 环上带有不同的取代基时都能得到不错的收率和中等 的立体选择性(表 2, Entries 1 4), 位阻较大的 1-菜基取 代的氯代底物相应的收率和立体选择性都有所降低(表 2, Entry 5). 而对于溴代烷烃, 在一 $30{ }^{\circ} \mathrm{C}$ 下反应时效果 很差, 收率很低, 延长反应时间至 $36 \mathrm{~h}$, 也不能提高转 化率. 因此将此类底物改在室温下反应，最终以良好的 收率, $20 \% \sim 30 \%$ 左右的 ee 值得到了相应的产物(表 2, Entries 6 11).

\section{3 反应机理的探讨}

基于 Glueck 等 $^{[8]}$ 在铂催化膦烷基化反应研究中提 出的反应机理, 和我们先前的研究工作: 钯催化二芳基 膦氢对缺电子烯酮的不对称加成反应中观测到 Pincer钯二苯基膦中间体的形成 ${ }^{[9 a]}$, 在此提出可能的反应过 程. 首先催化剂 4 与甲基苯基膦氢发生膦基转移生成具 有更高亲核性的甲基苯基膦钯中间体，然后对卤代烃发 生亲核取代反应生成膦产物配位的钯正离子中间体，在 体系中碳酸钾和甲基苯基膦氢的存在下释放出膦烷基 化产物(Scheme 1). 反应的立体化学过程可以由图 1 解 释. 由于钳形钯催化剂市位手性甲基的存在，导致膦原
表 1 甲基苯基膦氢的烷基化反应条件优化

Table 1 Optimization of reaction conditions for the alkylation reaction of methylphenylphosphine

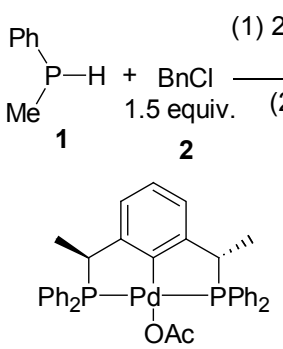

$(S, S)-4$
(1) $2 \mathrm{~mol} \%$ Cat. $4,1.5$ equiv. base

(2) $\mathrm{Me}_{2} \mathrm{~S} \cdot \mathrm{BH}_{3}$ solvent, $24 \mathrm{~h}$
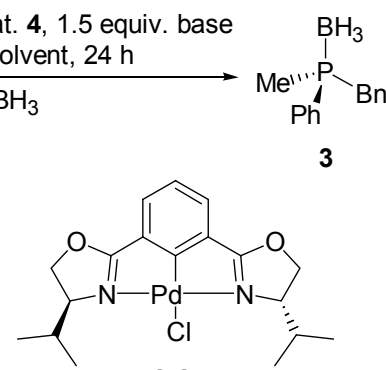

$(S, S)-5$

\begin{tabular}{|c|c|c|c|c|c|}
\hline Entry & Solvent & Base & $t /{ }^{\circ} \mathrm{C}$ & Yield $^{a} / \%$ & $e e^{b} / \%$ \\
\hline 1 & $\mathrm{CH}_{2} \mathrm{Cl}_{2}$ & $\mathrm{~K}_{2} \mathrm{CO}_{3}$ & r.t. & 83 & 32 \\
\hline $2^{c}$ & $\mathrm{CH}_{2} \mathrm{Cl}_{2}$ & $\mathrm{~K}_{2} \mathrm{CO}_{3}$ & r.t. & 24 & 0 \\
\hline 3 & THF & $\mathrm{K}_{2} \mathrm{CO}_{3}$ & r.t. & 91 & 33 \\
\hline 4 & Toluene & $\mathrm{K}_{2} \mathrm{CO}_{3}$ & r.t. & 96 & 32 \\
\hline 5 & $t-\mathrm{AmOH}$ & $\mathrm{K}_{2} \mathrm{CO}_{3}$ & r.t. & 85 & 33 \\
\hline 6 & DME & $\mathrm{K}_{2} \mathrm{CO}_{3}$ & r.t. & 87 & 36 \\
\hline 7 & $\mathrm{MeCN}$ & $\mathrm{K}_{2} \mathrm{CO}_{3}$ & r.t. & 83 & 38 \\
\hline 8 & Acetone & $\mathrm{K}_{2} \mathrm{CO}_{3}$ & r.t. & 87 & 40 \\
\hline 9 & EtOAc & $\mathrm{K}_{2} \mathrm{CO}_{3}$ & r.t. & 96 & 38 \\
\hline 10 & Nitroethane & $\mathrm{K}_{2} \mathrm{CO}_{3}$ & r.t. & 89 & 37 \\
\hline 11 & DMF & $\mathrm{K}_{2} \mathrm{CO}_{3}$ & r.t. & 93 & 45 \\
\hline 12 & DMF & $t$-BuOK & r.t. & 76 & 0 \\
\hline 13 & DMF & $\mathrm{NaOPh}$ & r.t. & 78 & 0 \\
\hline 14 & DMF & $\mathrm{NH}(i-\mathrm{Pr})_{2}$ & r.t. & 16 & 42 \\
\hline 15 & DMF & $\mathrm{NEt}_{3}$ & r.t. & 12 & 41 \\
\hline 16 & DMF & $\operatorname{NEt}(i-\operatorname{Pr})_{2}$ & r.t. & 12 & 42 \\
\hline 17 & DMF & $\mathrm{K}_{2} \mathrm{CO}_{3}$ & -30 & 92 & 53 \\
\hline 18 & DMF & $\mathrm{K}_{2} \mathrm{CO}_{3}$ & -55 & $<10$ & 59 \\
\hline $19^{d}$ & DMF & $\mathrm{K}_{2} \mathrm{CO}_{3}$ & -30 & $<5$ & - \\
\hline
\end{tabular}

${ }^{a}$ Isolated yields. ${ }^{b}$ Determined by HPLC with hexane/2-propanol; ${ }^{c} 5 \mathrm{~mol} \%$ cat. 5 was used. ${ }^{d}$ Without addition of Pd catalyst 4.

子上的两个苯环的空间取向不同，在钯周围构成了一个 手性空间，形成相对占优势的中间体 A 与 B 的平衡，与 苄氯反应得到 $(R)$-构型为主的产物 $\mathbf{3}$ (图 1). 反应取得中 等偏低的对映选择性可能是由于催化剂的手性空间仅 来源于膦原子上两个苯环的取向, 形成的立体空间位阻 区别不够大，不能很好的形成单一优势构象的中间体. 因此，探索合成具有较大立体位阻区别，手性环境更接 近膦原子的钳形钯催化剂, 来提高反应的立体选择性是 我们要进行的研究工作.

\section{3 结论}

本文作者使用 Pincer-钯催化剂研究了甲基苯基膦 氢对氯代，澳代烷烃的不对称取代反应. 以良好的收率， 中等的立体选择性获得了含膦手性中心的膦化合物. 


\section{表 2 底物拓展}

Table 2 Extension of the substrate scope

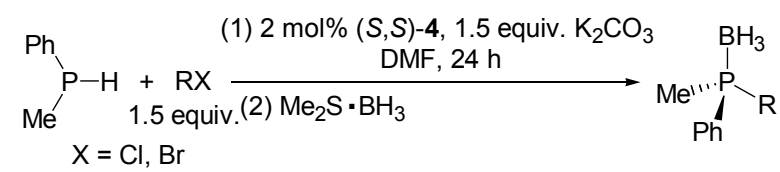

\begin{tabular}{|c|c|c|c|c|}
\hline Entry & Substrate & $t /{ }^{\circ} \mathrm{C}$ & Yield $^{a} / \%$ & $e e^{b, c} / \%$ \\
\hline 1 & $\mathrm{BnCl}$ & -30 & 92 & 53 \\
\hline 2 & $p-\mathrm{MeC}_{6} \mathrm{H}_{4} \mathrm{CH}_{2} \mathrm{Cl}$ & -30 & 95 & 52 \\
\hline 3 & $o-\mathrm{MeC}_{6} \mathrm{H}_{4} \mathrm{CH}_{2} \mathrm{Cl}$ & -30 & 87 & 45 \\
\hline 4 & $p-\mathrm{ClC}_{6} \mathrm{H}_{4} \mathrm{CH}_{2} \mathrm{Cl}$ & -30 & 95 & 56 \\
\hline 5 & 1-NaphCH${ }_{2} \mathrm{Cl}$ & -30 & 64 & 34 \\
\hline 6 & & r.t. & 81 & 34 \\
\hline 7 & & r.t. & 92 & 25 \\
\hline 8 & & r.t. & 93 & 25 \\
\hline 9 & B & r.t. & 75 & 35 \\
\hline 10 & $\mathrm{Br}$ & r.t. & 82 & 33 \\
\hline $11^{d}$ & $\mathrm{Br}^{-}$ & r.t. & $70^{e, f}$ & 24 \\
\hline
\end{tabular}

${ }^{a}$ Isolated yields. ${ }^{b}$ Determined by HPLC with hexane/2-propanol. ${ }^{c}$ The absolute configurations of products were determined to be $R$ by comparison of the specific optical rotation of the product (Entry 1) with the value reported in literature (see Supporting Information for details); ${ }^{d} 0.5$ equiv. dibromide was used. ${ }^{e}$ Combined yields of racemate and mesomer. ${ }^{f}$ The ratio of racemate to mesomer $(1.06: 1)$ was determined by HPLC analysis.

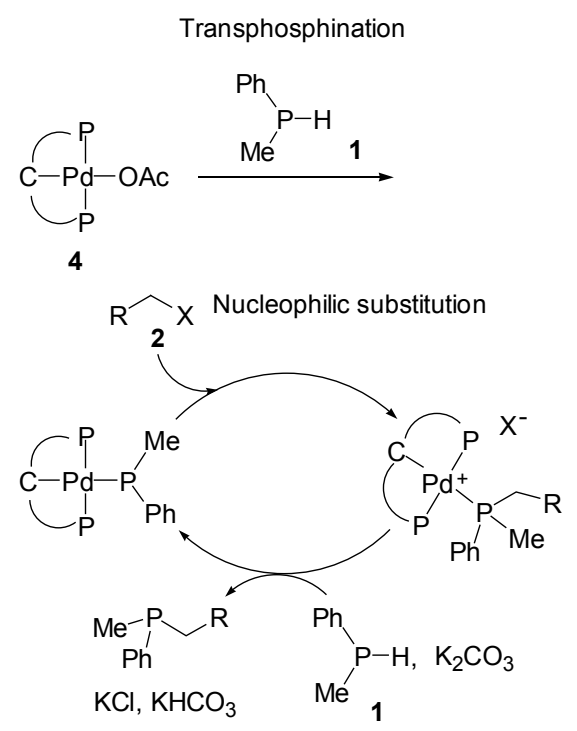

Scheme 1

辅助材料(Supporting Information) 实验步骤、产物数 据及谱图。这些材料可以免费从本刊网站 (http://sioc-journal.cn/)上下载.
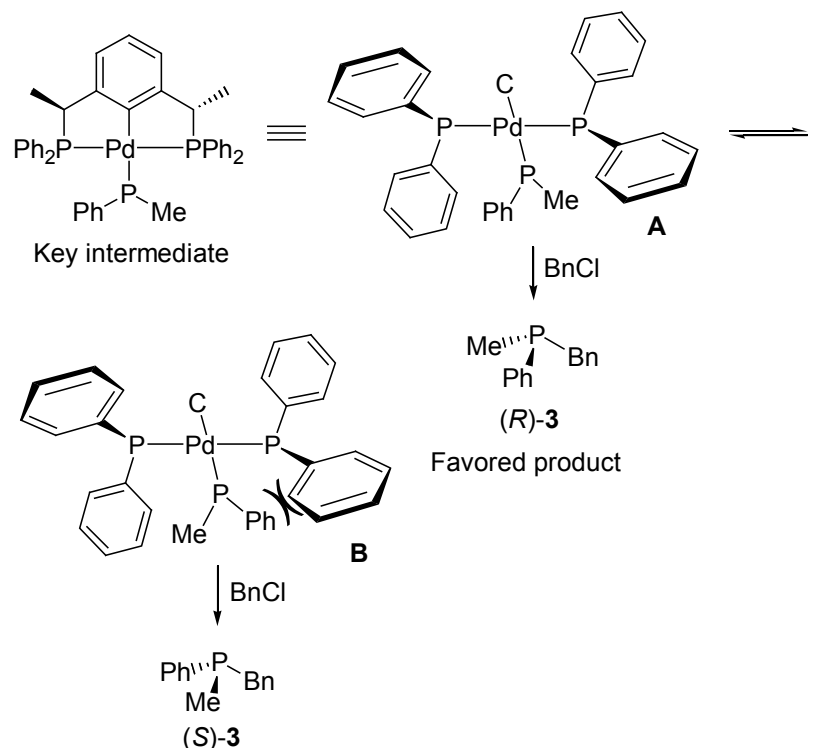

$\mathrm{Me}_{\mathrm{Ph}}^{\prime \prime \prime} \mathrm{Pn}$

$(R)-3$

Favored product

Unfavored product

图 1 可能的立体化学过程

Figure 1 Proposed stereochemical pathway

\section{References}

[1] Grabulosa, A. P-stereogenic Ligands in Enantioselective Catalysis, Cambridge, UK, 2011.

[2] Horner, L.; Winkler, H.; Rapp, A.; Mentrup, A.; Hoffmann, H.; Beck, P. Tetrahedron Lett. 1961, 2, 161.

[3] (a) Knowles, W. S.; Sabacky, M. J. Chem. Commun. 1968, 1445. (b) Horner, L.; Siegel, H.; Büthe, H. Angew. Chem. Int. Ed. 1968, 7, 942.

[4] For reviews on catalytic enantioselective synthesis of chiral phosphines, see: (a) Glueck, D. S. Synlett 2007, 2627.

(b) Glueck, D. S. Chem.-Eur. J. 2008, 14, 7108.

(c) Harvey J. S.; Gouverneur, V. Chem. Commun. 2010, 7477.

(d) Zhao, D.; Wang, R. Chem. Soc. Rev. 2012, 41, 2095.

[5] (a) Lebel, H.; Morin, S.; Paquet, V. Org. Lett. 2003, 5, 2347.

(b) Wiketelius, D.; Johansson, M. J.; Luthman, K.; Kann, N. Org. Lett. 2005, 7, 4991.

(c) Gammon, J. J.; Canipa, S. J.; O'Brien, P.; Kelly, B.; Taylor, S. Chem. Commun. 2008, 3750.

(d) Nishida, G.; Noguchi, K.; Hirano, M.; Tanaka, K. Angew. Chem. Int. Ed. 2008, 47, 3410 .

(e) Harvey, J. S.; Malcolmson, S. J.; Dunne, K. S.; Meek, S. J.; Thompson, A. I.; Schrock, R. R.; Hoveyda, A. H.; Gouverneur, V. Angew. Chem. Int. Ed. 2009, 48, 762.

(f) Liu, S.; Zhang, Z.; Xie, F.; Butt, N.; Sun, L.; Zhang, W. Tetrahedron: Asymmetry 2012, 23, 329.

[6] (a) Kovacik, I.; Wicht, D. K.; Grewal, N. S.; Glueck, D. S.; Incarvito, C. D.; Guzei, I. A.; Rheingold, A. L. Organometallics 2000, 19,950 .

(b) Scriban, C.; Kovacik, I.; Glueck, D. S. Organometallics 2005, 24, 4871 .

(c) Join, B.; Mimeau, D.; Delacroix, O.; Gaumont, A.-C. Chem. Commun. 2006, 3249.

(d) Huang, Y.; Pullarkat, S. A.; Li, Y.; Leung, P.-H. Inorg. Chem. 2012, 51, 2533.

[7] For examples of catalytic asymmetric synthesis of chiral phosphines through arylation of substituted phosphines, see:

(a) Moncarz, J. R.; Laritcheva, N. F.; Glueck, D. S. J. Am. Chem. 
Soc. 2002, 124, 13356.

(b) Korff, C.; Helmchen, G. Chem. Commun. 2004, 530.

(c) Blank, N. F.; Moncarz, J. R.; Brunker, T. J.; Scriban, C.; Anderson, B. J.; Amir, O.; Glueck, D. S.; Zakharov, L. N.; Golen, J. A.; Incarvito, C. D.; Rheingold, A. L. J. Am. Chem. Soc. 2007, 129, 6847.

(d) Chan, V. S.; Bergman, R. G.; Toste, F. D. J. Am. Chem. Soc. 2007, 129, 15122.

[8] For some examples of catalytic asymmetric synthesis of chiral phosphines through alkylation of substituted phosphines, see:

(a) Chan, V. S.; Stewart, I. C.; Bergman, R. G.; Toste, F. D. J. Am. Chem. Soc. 2006, 128, 2786.

(b) Scriban, C.; Glueck, D. S. J. Am. Chem. Soc. 2006, 128, 2788.

(c) Scriban, C.; Glueck, D. S.; Golen, J. A.; Rheingold, A. L. Organometallics 2007, 26, 1788.

(d) Anderson, B. J.; Glueck, D. S.; DiPasquale, A. G.; Rheingold, A.

L. Organometallics 2008, 27, 4992.

(e) Anderson, B. J.; Guino-o, M. A.; Glueck, D. S.; Golen, J. A.; DiPasquale, A. G.; Liable-Sands, L. M.; Rheingold, A. L. Org. Lett. 2008, 10, 4425 .

(f) Chan, V. S.; Chiu, M.; Bergman, R. G.; Toste, F. D. J. Am. Chem. Soc. 2009, 131, 6021.

(g) Chapp, T. W.; Glueck, D. S.; Golen, J. A.; Moore, C. E.; Rhein- gold, A. L. Organometallics 2010, 29, 378.

(h) Chapp, T. W.; Schoenfeld, A. J.; Glueck, D. S. Organometallics 2010, 29, 2465.

(i) Guino-o, M. A.; Zureick, A. H.; Blank, N. F.; Anderson, B. J.; Chapp, T. W.; Kim, Y.; Glueck, D. S.; Rheingold, A. L. Organometallics 2012, 31, 6900..

[9] (a) Feng, J.-J.; Chen, X.-F.; Shi, M.; Duan, W.-L. J. Am. Chem. Soc. 2010, 132, 5562 .

(b) Du, D.; Duan, W.-L. Chem. Commun. 2011, 47, 11101.

(c) Chen, Y.-R.; Duan, W.-L. Org. Lett. 2011, 13, 5824.

(d) Feng, J.-J.; Huang, M.; Lin, Z.-Q. Duan, W.-L. Adv. Synth. Catal. 2012, 354, 3122.

(e) Huang, M.; Li, C.; Duan, W.-L.; Xu, S. Chem. Commun. 2012, 48,11148 .

[10] For reviews on pincer metal complexes, see:

(a) Albrecht, M.; van Koten, G. Angew. Chem. Int. Ed. 2001, 40, 3750 .

(b) Selander, N. Szabó, K. J. Chem. Rev. 2011, 111, 2048.

[11] For synthesis and application of the chiral PCP-PdCl complex, see:

(a) Longmire, J. M.; Zhang, X. Tetrahedron Lett. 1997, 38, 1725.

(b) Longmire, J. M.; Zhang, X.; Shang, M. Organometallics 1998, 17,4374 .

(Li, L.; Fan, Y.) 